\title{
Investigation of Noble Metals Colloidal Systems Formed by Laser Synthesis at Air
}

\author{
Victor Goncharov, Konstantin Kozadaev, and Dzmitry Shchehrykovich
}

A.N. Sevchenko Research Institute of Applied Physical Problems of Belarusian State University, Kurchatov Street 7, 220108 Minsk, Belarus

Correspondence should be addressed to Konstantin Kozadaev, kozadaeff@mail.ru

Received 24 October 2011; Revised 28 May 2012; Accepted 4 June 2012

Academic Editor: Yeshoshua Kalisky

Copyright ( 92012 Victor Goncharov et al. This is an open access article distributed under the Creative Commons Attribution License, which permits unrestricted use, distribution, and reproduction in any medium, provided the original work is properly cited.

The present work is dedicated to the development of formation and diagnostics methods of water colloids of noble metals (Au, $\mathrm{Au}, \mathrm{Pt}$ ). As anoble nanoparticles formation method, the laser synthesis at air conditions is proposed. By the implantation of noble nanoparticles into water media, the colloidal systems of noble metals can be obtained. For the aims of investigation of noble colloid parameters, the complex diagnostics method is used. Such approach deals with direct methods (scanning electron microscopy and the characteristic radiation registration) and indirect methods (absorption spectroscopy and extinction modeling by Mie theory).

\section{Introduction}

Currently, nanotechnologies and nanoengineering are one of the most promising directions in the development of modern science. Great practical interest to nanoobjects is caused by the presence of a number of specific properties corresponding to them (physical, chemical, biological), which are not peculiar to massive objects, consisting of the same material. This, for example, allows to modify the traditional media by metal nanostructures, thereby gaining strength, durability, abrasion resistance, and so forth.

Now there are many industrial technologies of metal nanoparticles production. All of them can be divided into the following groups of methods: elementary wet lab (subsidence, revivification), synthesis of gaseous and solid phases, chemical methods of homogenization, the methods based on the use of one- and two-dimensional reactors, methods based on such phenomena as self-organization and self-assembly, as well as various types of lithography [1]. All of the abovesaid methods of formation of metal nanoparticles have their own advantages and disadvantages and can be used to solve certain technological problems.

As an example of one of such tasks, the receiving of colloid solutions of noble metals nanoparticles can be mentioned. These colloids are widely used for the needs of petrochemistry, organic chemistry, polymer chemistry, as well as in light industry [2].

Fundamental features of noble metals (such as inertia, relative refractoriness, and high specific density) do not allow the proper use of all the above-said methods of synthesis. As one of the promising methods for formation of noble metals colloids, the method of laser synthesis at air can be applied. The main advantages of this technology are simplicity of technical implementation, an opportunity to perform operational control over the synthesis process, and a relatively short acquisition time for the formation of collids. The essence of this approach is discussed in more details in articles $[3,4]$. The present paper deals with formation noble metals nanoparticles $(\mathrm{Ag}, \mathrm{Au}$, and $\mathrm{Pt})$ at air conditions and investigation of their spectral-morphological characteristics at the case of water media.

\section{Method}

Based on the earlier studies of the atmospheric features of the metal laser erosion by submicrosecond ( $100 \mathrm{~ns}$ ) highintensity light pulses $(\lambda=1064 \mathrm{~nm})$ in the air, it has become well-known that $\sim 50 \mathrm{~ns}$ after the laser action (the time comparable to the duration of the rise front of current pulse) 
the plasma jet (or Erosion Laser Torch-ELT) is formed in the surface area of the target [5]. ELT expands in the direction of the external environment, consuming due to the inverse braking effect almost all the energy of the back of the front of the acting optical pulse and, consequently, significantly warms up. A typical propagation velocity of the plasma jet at the initial moment of its formation is $7-20 \mathrm{~km} / \mathrm{s}$ depending on the material of the metal target [6].

After the fall of the laser pulse intensity, the fairly slow cooling of the torch starts mainly due to adiabatic expansion of the plasma. During the passing of this process in the spatial structure of the ELT, the density fluctuations of the torch vapor appear. Such fluctuations in their turn give rise to the condensation processes. At the same time after $2-2.5 \mu \mathrm{s}$ from the beginning of the laser action, the vapor pressure inside the torch becomes equal to the external air pressure, so the plasma expanding stops. Thereby the prerequisites for a stable condensation of torch vapors have taken place. Due to this fact in the surface region of the target, the conditions for fine particles formation are created. Studies of this regime of laser erosion indicate [3] that the drop formation process lasts for 300-400 $\mu$ s after the laser action and ultimately leads to the observing of the target material nanoparticles flow from the surface area of the laser target. Studies in $[3,7]$ evaluate the basic parameters of the evolving ultrafine phase, according to them the size of particles belongs to the nanometer range of $30-140 \mathrm{~nm}$ and shows the relatively high level of concentration $\left(10^{11}-10^{13} \mathrm{~cm}^{-3}\right)$. According to research works $[8,9]$, the technique of laser synthesis can penetrate the metal nanoparticles formed in the ELT in optically clear solution, thereby forming colloidal systems of the treated metal material.

In the course of investigation of the formation conditions for the metal liquid-drop phase ( $\mathrm{Ag}, \mathrm{Zn}, \mathrm{Pb}, \mathrm{Ni}, \mathrm{Cu}$ ) at all cases of target material, the effect of improving of the conditions for metal drops appearing with the increasing of the surface roughness of the target was showed. In this case, as a rule, the particles of more refractory metals are of the smaller size and have high concentration compared to the low-melting ones under otherwise equal conditions of laser irradiation. Nanoparticles of the target material may be found in the surface area up to $500 \mu \mathrm{s}$ after the laser action, having their average velocity of $50-100 \mathrm{~m} / \mathrm{s}$ depending on the type of metal. As a result, investigations have also shown that the factor of the reflection coefficient of laser radiation by metal targets does not play a determining role in the process of laser erosion and further drops formation, because regardless of its initial value, due to the intense optical radiation the macrolayer of phase transition "solid-vapor" is formed in which the absorption increases significantly. The following parameters are dominant in this case: the current intensity of the irradiation, melting and evaporation temperature, the latent heat of melting, and evaporation of the material [3].

As a general rule [6] with a single laser pulse of the high power density a sufficiently small number of particles is formed (total mass of particles in the condensed phase bearable during the single-pulse treatment is tens of micrograms, depending on the type of metal and treatment

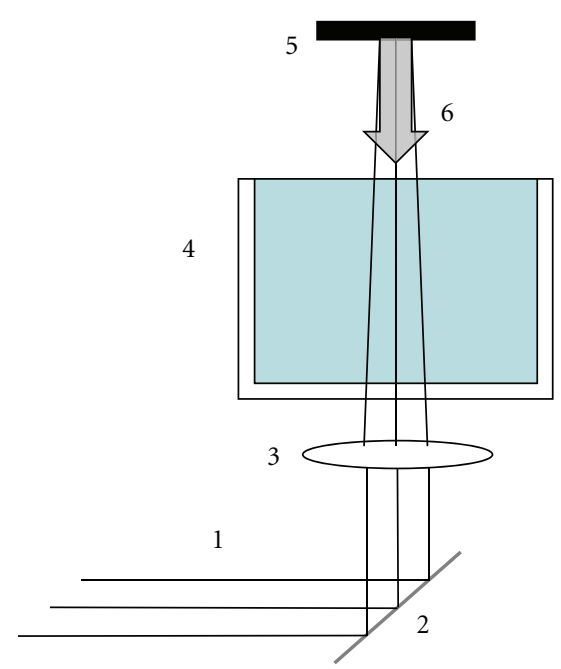

Figure 1: Experimental setup.

conditions). Therefore, to obtain a sufficiently concentrated colloidal solutions of metals it is advisable to use a sequence of laser pulses which can be implemented using modern laser technology in the frequency mode.

Studies $[3,10]$ which try to evaluate the effectiveness of the condensed nanosized metal phase formation in the case of multiple laser irradiation without changing localization of beam observe the effect of smoothing the original topography of the target was discovered, which, in turn, leads to worsening conditions for the formation of condensed phase of metals. Regardless of the initial surface roughness (roughness size up to $100 \mu \mathrm{m}$ ) after four or five pulses of incident radiation the conditions for the formation of the condensed phase of the target material do not differ much from the erosion of smooth targets (with an average size of unevenness $\sim 3 \mu \mathrm{m}$ ). From this standpoint it is expedient to constantly change the localization of the treatment spot to maintain a constant level of the volume formed by nanoscale phase.

\section{Experiment}

At the present work for the formation of colloidal solutions of nanoparticles of noble metals Nd: YAG $(\lambda=1064 \mathrm{~nm})$ laser was used which provides a pulse duration of $20 \mathrm{~ns}$ and an average pulse energy of $200 \mathrm{~mJ}$, which when focused into a spot with $d=1 \mathrm{~mm}$ may help obtain the power density of $\sim 1 \mathrm{GW} / \mathrm{cm}^{2}$. The pulse repetition rate was $5 \mathrm{~Hz}$. The typical exposure time for optically dense samples of metal colloids has been chosen to be $15 \mathrm{~min}$ for one sample $(v=120 \mathrm{~mL})$.

The process of synthesis occurred in the air, followed by penetration of the formed noble metals nanoparticles into the water medium. Experimental scheme of the laser synthesis method is deposited at the Figure 1.

The intense laser irradiation 1 is turned by the angle 90 with interference mirror 2. Then the laser beam is focused with the help of lens 3 and comes through the pan with a medium of penetration to metal target 5 . The correction of additional focusing caused by parallel-sided lay of liquid 
was done by experimental way. The condensed phase of target material (formed within the erosion laser jet) directly permeates through opened liquid surface. Each of the different targets 5 represents massive plates of silver, gold, and platinum, chemical homogeneity of which has been confirmed by the government certification.

For the investigation of metal nanoparticles properties in optical media it is appropriate to apply the complex of direct and indirect methods, in order to obtain the most complete information about the condition and properties of metals nanophases [9]. For direct methods of diagnostics of ultrafine metallic phase parameters the following techniques were used in this study: scanning electron microscopy (SEM NEON 40 Karl Zeiss) and registration of the characteristic spectrum of microscopic objects excited by a narrowly focused electron beam (electron probe). The indirect methods are presented by absorption spectroscopy of surface plasmon resonance (SPR) and by a comparison of the actual shape of the SPR band with model spectral dependences of efficient factor of extinction, constructed according to the Mie theory.

Modeling the efficient factor of extinction of metallic colloidal systems in order to compare it with the real spectra of SPR is a wellknown method of approximate estimation of the parameters of the ultrafine metal phase in optically transparent media [11]. In order to determine the spectral shape efficient factor of extinction the approach was used based on the theory of interaction of electromagnetic field with small spherical particles, proposed by Mie $[12,13]$. This theory is widely used for modeling of the optical characteristics of low-dimensional objects $[14,15]$, it is based on the decomposition of electromagnetic fields interacting with a small particle on vector spherical harmonics, with subsequent calculation of the coefficients of this distribution. The main positions of the approach are presented in [16]. For numerical calculations MiePlot 4207 software was used. The program allows the dependences of extinction effectiveness factors, absorption and scatter on the wavelength of incident planar radiation front to be calculated taking into account the spectral dependences of imaginary and real parts of the refractive index of particle material and aqueous environment. A normal spherical particle-size distribution with different values of the scatter is takeng as a model.

\section{Discussion of the Results}

Using the technique of laser synthesis of metal nanoparticles, mentioned at the previous section three experimental samples were set containing a colloidal system of silver, gold and platinum in the water medium. In this case the samples were optically homogeneous and as a result of the SPR effect they were painted as follows: the silver colloid was rich yellow brown gold colloid was rich pink ruby. The platinum colloidal solution did not have any clearly defined coloring, having a visible translucent gray tint, which indicates the absence of severe narrowband extinction in the visible area.

Then the experimental samples were subjected to an investigation using the SEM techniques, for which small amounts $(\sim 1 \mathrm{~mL})$ of each colloid were deposited on a carbon film, the water slowly evaporated, and the nanoparticles of metal gradually deposited on the substrate. After this, the formed products of metal colloids were evacuated and subjected to SEM. SEM images for each type of colloid is placed in Figures 2(a), 2(b) and 2(c) (silver, gold, and platinum, resp.). In all three images there are spherical nanoobjects, which differ considerably in size. A common feature of the images under consideration is a grouping of the smaller objects around the large ones, which might suggest the mechanism of clustering of particles visualized conglomerates.

At the same time during the processing of a large array of such images it becomes apparent that in the local absence of large objects smaller particles are reluctant to form an array, trying to evenly distribute the area of the substrate. Thus the visualized combining of particles often cannot be representative for the determination of an average particle size of a real colloid and of the characteristics of their size distribution, in fact, only giving the information about the predominant form of produced nanoobjects.

During the next stage of the study the revealed conglomerates of nanosized objects were subjected to excitation by a narrowly focused electron beam (electron probe) in order to initiate and to further register the characteristic radiation (X-rays). The results of this procedure are shown in Figures 2(d), 2(e) and 2(f) for silver, gold and platinum, respectively. When considering these spectra one can conclude that the visualized nanoparticles are chemically homogeneous and consist entirely of the target material. The detection of minor traces of foreign elements is due to the way of making preparations and to the type of substrate used.

The results of absorption spectroscopy of the samples of colloidal solutions are placed in Figures 2(g), 2(h), and 2(i) (curve 1) for silver gold, and platinum, respectively. The spectra of silver and gold colloids form the distinct peaks of extinction with maxima in $403 \mathrm{~nm}$ and $520 \mathrm{~nm}$ respectively which is caused by the effect of SPR and which explains a certain coloring of the experimental samples [17]. Colloid which contains nanoparticles of platinum, is characterized by wide "spectral wings" ascending in the short-wavelength area which is in good agreement with the parameters of platinum colloid formed by chemical methods [18].

Modeling spectral dependences of the efficient factor of extinction, which was approximated according to the Mie theory, are shown in Figures 2(g), 2(h) and 2(i) (curve 2) for silver, gold and platinum, respectively. The modeling parameters (mean diameter and variance of the distribution on the diameter values of the solid metal spheres) for each of the metal are chosen in such way as to ensure the maximum compliance with the actual contours of extinction (curve 1 in Figures 2(g), 2(h) and 2(i)).

According to the results of modeling the efficient factor of extinction we can state that the evaluation of average particle diameter in real colloids of silver, gold, and platinum are $60 \mathrm{~nm}, 40 \mathrm{~nm}$, and $25 \mathrm{~nm}$, respectively, with the dispersion of particle size distribution of $25 \%$ for all studied metals. The values of the optical characteristics of these metals are taken from [19]. This value is typical for the dispersion of 


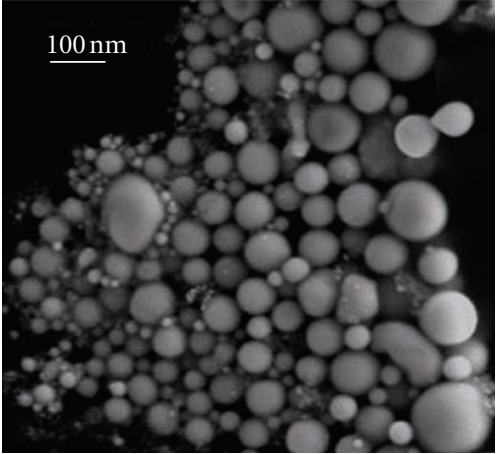

(a)

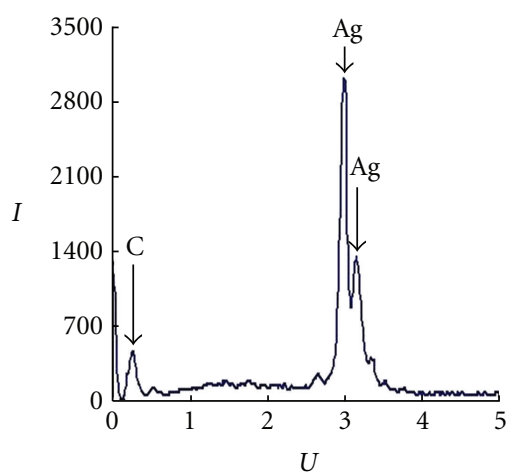

(d)

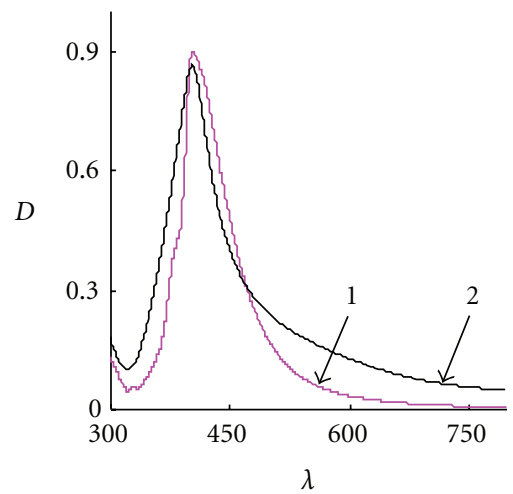

(g)

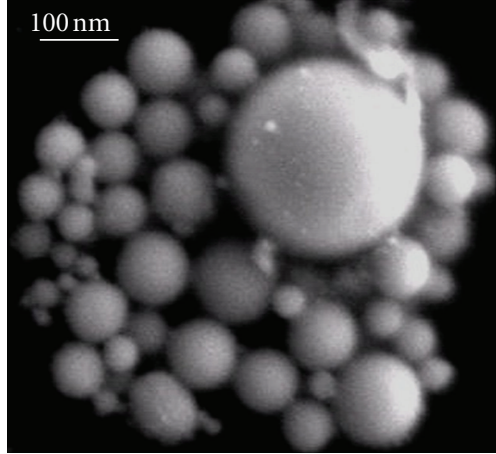

(b)

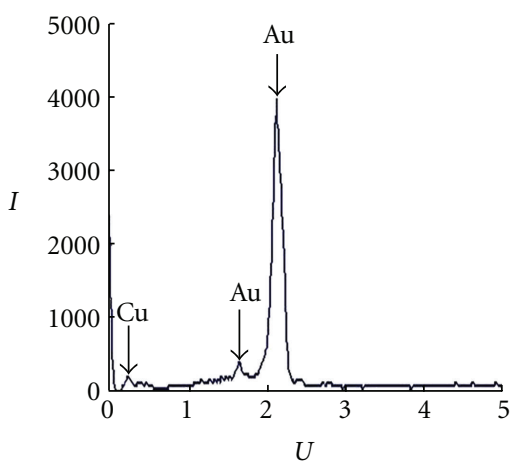

(e)

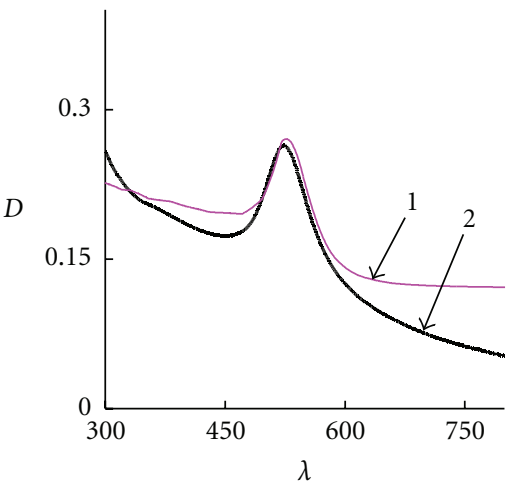

(h)

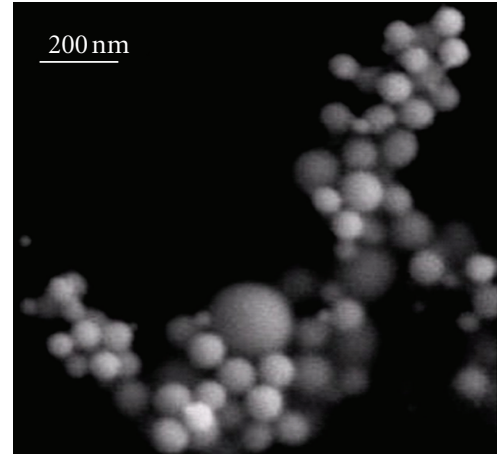

(c)

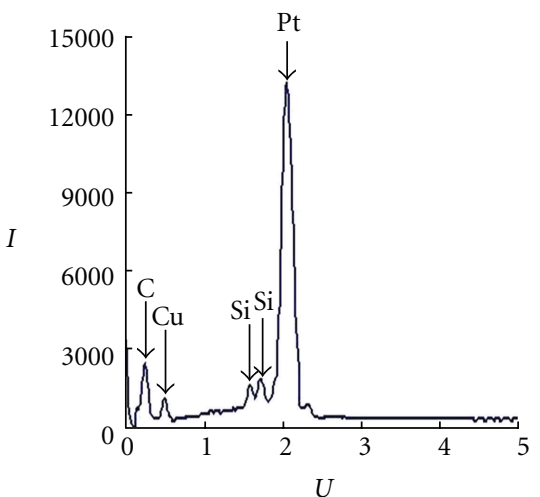

(f)

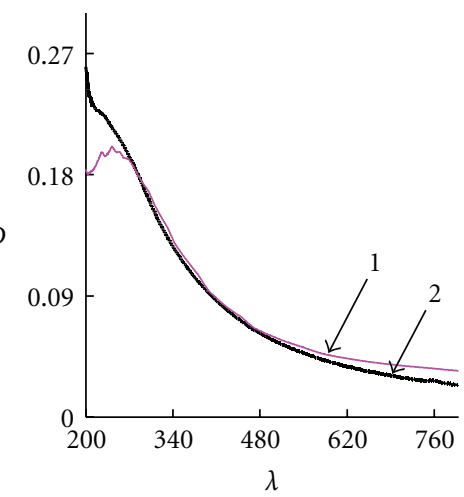

(i)

FIGURE 2: The results of the studies of experimental models of metals aquatic colloids: SEM image of particles of (a) silver, (b) gold, and (c) platinum deposited on a substrate; range of the characteristic radiation of particles in electron beam of (d) silver, (e) gold, and (f) platinum; SPR band spectrum of (g) silver, (h) gold, and (i) platinum (1-a real, 2-model).

ultrafine metallic systems formed by condensation processes [9]. In this case the earlier regularities mentioned above can be illustrated: the particles of a refractory gold, and platinum characterized by smaller average size, compared with silver.

Based on the results of complex studies of experimental models of colloids of noble metals $(\mathrm{Au}, \mathrm{Au}, \mathrm{Pt})$ the following conclusions can be drawn:

(i) With the help of the technique of laser synthesis the implementation of an effective process of formation of colloidal solutions of noble metals $(\mathrm{Au}, \mathrm{Au}$, and $\mathrm{Pt}$ ) is possible. This approach has several advantages: simplicity of technical implementation, an opportunity to manage the process of synthesis, universality with respect to the type of metal, as well as a relatively short time of exposure for the formation of solutions.

(ii) Direct methods for the study of isolated nanoobjects (SEM and electron probe) in this case can only form a qualitative understanding of the system under study, since the results of the study are strongly influenced by processes occurring during the dissection of samples. However despite this disadvantage, these techniques can reliably determine the morphological 
parameters of nano-objects (mainly the shape and composition), which are also important characteristics of a particular metal colloidal system.

(iii) To quantify the main parameters of the colloidal system (average particle diameter and dispersion of their size distribution), it is advisable to use a method of modeling the efficient factor of extinction of metallic colloidal systems in order to compare with the real spectra of SPR.

\section{Conclusion}

Based on the technique of laser synthesis in an air atmosphere an industrial technology of formation of the noble metals nanoparticles flow can be developed. The main advantages of this technological approach are the simplicity of technical realization of the process, low-cost production, and high rate of synthesis of metal nanoparticles. When used as a medium for the introduction of water the formation of water colloidal solutions of the processed metals with high optical density is possible. For the diagnosis of the spectral and morphological parameters of the formed colloids it is expedient to use set of direct (SEM and registration of the characteristic spectrum upon excitation by an electron beam) and indirect (SPR spectroscopy and modeling of the extinction of the colloid based on the Mie theory) methods.

\section{Acknowledgment}

The authors wish to thank OPTEC LLC for financial support of the present work under the international OPTEC Grant Program.

\section{References}

[1] C. Raab, M. Simko, U. Fiedeler, M. Nentwich, and A. Gaszso, "Herstellung von nanopartikeln und nanomaterialien," Nanotrustdossiers, vol. 206, pp. 1-4, 2008.

[2] C. P. Poole Jr. and F. J. Owens, Introduction to Nanotechnology, Wiley, John \& Sons, 1 edition, 2003.

[3] V. K. Goncharov and K. V. Kozadaev, "Formation of the condensed phase of metals exposed to submicrosecond laser pulses," Journal of Engineering Physics and Thermophysics, vol. 83 , no. 1 , pp. 90-95, 2010.

[4] V. K. Goncharov, D. R. Ismailov, K. V. Kozadaev, and M. V. Pusyrev, "Optical memory \& neural networks," Information Optics, vol. 4, pp. 254-262, 2007.

[5] V. K. Goncharov, K. V. Kozadaev, M. V. Puzyrev, and V. M. Stetsik, "Dynamics of parameters of an erosion torch formed under the action of submicrosecond laser radiation on a zinc target," Journal of Engineering Physics and Thermophysics, vol. 82, no. 4, pp. 630-634, 2009.

[6] V. K. Goncharov, K. V. Kozadaev, and D. V. Schegrikovich, "Start of condensation in erosional jets of metals subjected to highly intense submicrosecond laser action," Journal of Engineering Physics and Thermophysics, vol. 84, no. 4, pp. 781786, 2011.

[7] V. K. . Goncharov, K. V. Kozadaev, and M. V. Puzyrev, "The influence of ND laser irradiation parameters on dynamics of metal condensed phase propagating near target," in
Engineering Physics and Mechanics: Analyses, Prediction and Applications, M. Sosa and J. Franco, Eds., pp. 441-471, 2010.

[8] V. K. Goncharov, K. V. Kozadaev, M. V. Puzyrev, and V. I. Popechits, "Combination method for monitoring the characteristics of aqueous suspensions of metallic nanoparticles," Journal of Applied Spectroscopy, vol. 75, no. 6, pp. 892-897, 2008.

[9] V. K. Goncharov, K. V. Kozadaev, and D. I. Shiman, "Formation and complex investigation of spectral-morphologic parameters of Ag nanoscale phase in polymer film," Journal of Applied Spectroscopy, vol. 77, no. 5, pp. 675-679, 2010.

[10] K. V. Kozadaev, V. K. Goncharov, and D. V. Shchehrykovich, "The investigation of two-phase plasma fluxes of silver," Publications of The Astronomical Observatory of Belgrade, vol. 89, pp. 139-141, 2010.

[11] L. A. Bumm, NanoLab. Mie Theory and Extinction Spectra, University of Oklahoma, Oklahoma, Okla, USA, 2007.

[12] H. C. Hulst van de, Light Scattering by Small Particles, Dover, 1981.

[13] C. F. Bohren and D. R. Huffman, Absorption and Scattering of Light by Small Particles, John Wiley \& Sons, 1998.

[14] R. A. Dynich and A. N. Ponyavina, "Effect of metallic nanoparticle sizes on the local field near their surface," Journal of Applied Spectroscopy, vol. 75, no. 6, pp. 832-838, 2008.

[15] K. V. Kozadaev, "Diagnostics of aqueous colloids of noble metals by extinction modeling based on mie theory," Journal of Applied Spectroscopy, vol. 78, no. 5, pp. 742-748, 2011.

[16] U. Kreibig and M. Volmer, Optical Properties of Metal Clusters, Springer, New York, NY, USA, 1993.

[17] V. A. Bogatyrev, L. A. Dykman, and N. G. Khlebtsov, "Methods for the synthesis of nanoparticles with a plasmon resonance" (Russian), SSU named after N. G. Chernyshevsky, Saratov, Russia, 2009.

[18] B. G. Yershov, "Nanoparticles of Metals in water solutions: electronic, optical and catalytical properties," Russian Journal of Chemistry, vol. XLV, no. 3, pp. 20-30, 2001.

[19] E. D. Palik, Handbook of Optical Constants of Solids, Academic Press, Orlando, Fla, USA, 1985. 

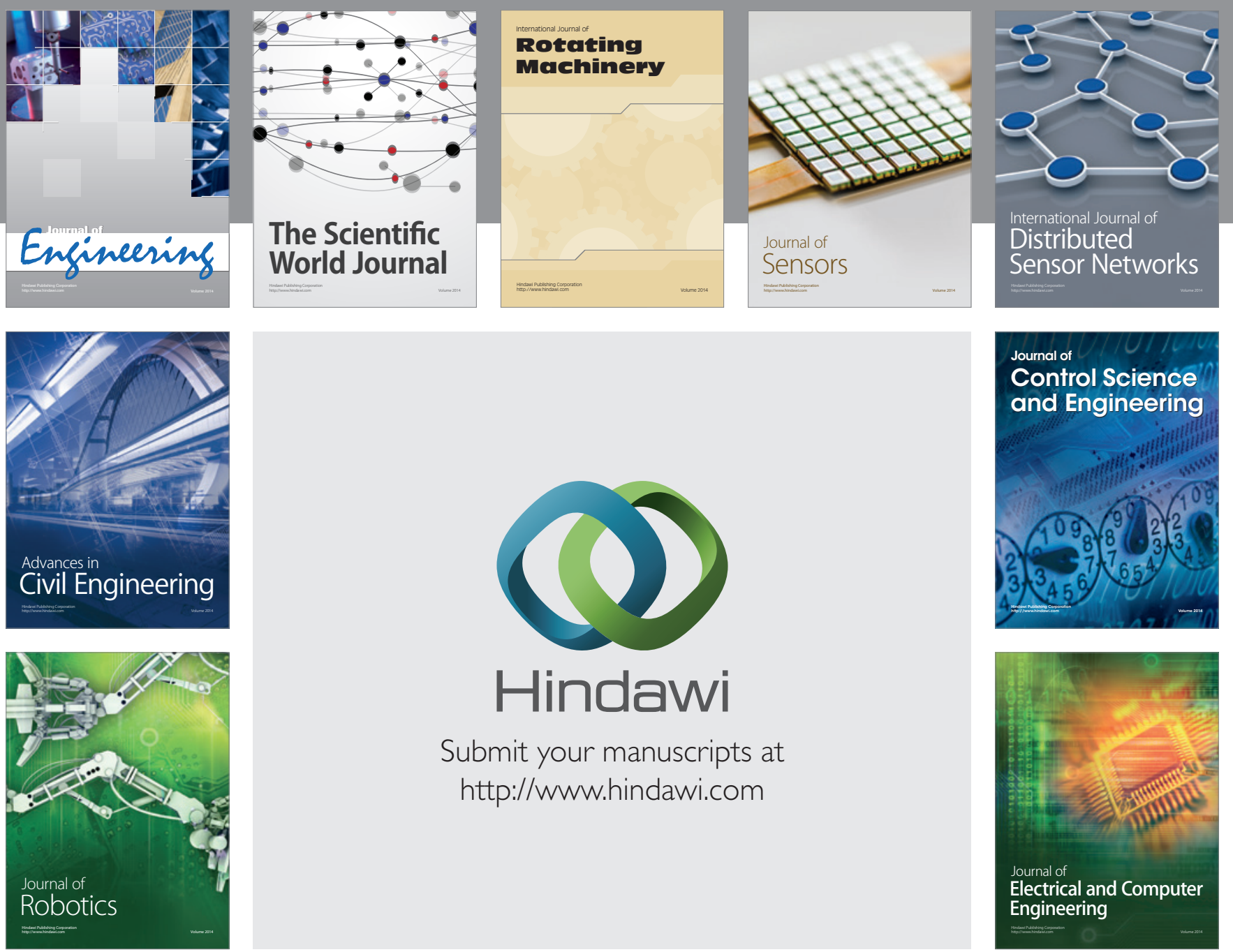

Submit your manuscripts at

http://www.hindawi.com
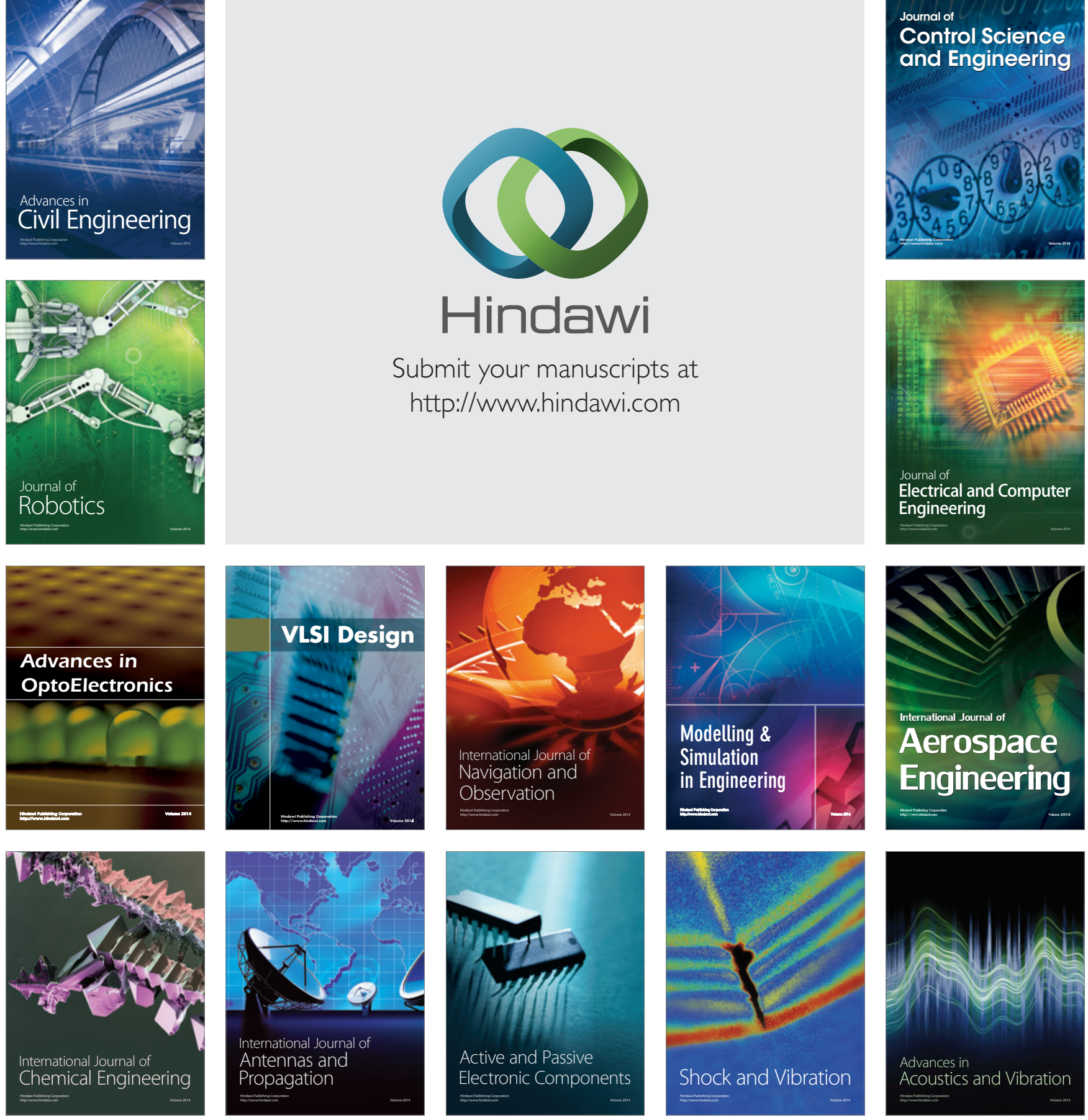